\title{
Smad2/3/4 Pathway Contributes to TGF- $\beta$-Induced MiRNA-181b Expression to Promote Gastric Cancer Metastasis by Targeting Timp3
}

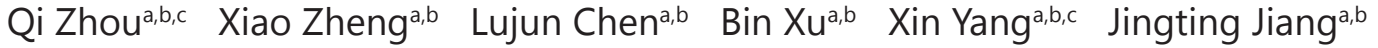 \\ Changping $\mathrm{Wu}^{\mathrm{a}, \mathrm{b}, \mathrm{c}}$
}

aDepartment of Tumor Biological Treatment, Changzhou, bJiangsu Engineering Research Center for Tumor Immunotherapy, Changzhou, 'Department of Oncology, the Third Affiliated Hospital of Soochow University, Changzhou, China

\author{
Key Words \\ Gastric cancer $•$ TGF- $\beta \cdot$ EMT $・$ MiRNA-181b $\cdot$ Smad4 $・$ Timp3
}

\begin{abstract}
Background/Aims: Transforming growth factor beta (TGF- $\beta$ ) plays a major role in tumorigenesis. MicroRNA-181b (miRNA-181b) is a multifaceted miRNA that has been implicated in many cellular processes such as cell fate determination and cellular invasion. This study aimed to confirm the relationship of miRNA-181b and the TGF- $\beta$-Smad2/3/4 pathway with the induction of the epithelial-to-mesenchymal transition (EMT) in gastric cancer. Methods: This study investigated the ability of TGF- $\beta$ to induce migration by wound healing and transwell invasion assays in human gastric cancer cell lines. miRNA expression was altered using miRNA-181b mimic and inhibitor in the same system. Expression of miRNA$181 \mathrm{~b}$, the hypothetical target gene Timp3 and EMT-related markers were analyzed by realtime real-time quantitative RT-PCR. Immunoblotting was used to investigate the levels of phospho-Smad2 and Smad4. Dual-luciferase reporter assays were performed to confirm the direct binding of miRNA-181b to Timp3. Results: miRNA-181b was significantly upregulated in response to TGF- $\beta$ treatment in gastric cancer cell lines. Overexpression of miR-181b mimic induced an in vitro EMT-like change to a phenotype similar to that following TGF- $\beta$ treatment alone and was reversed by miRNA-181b inhibitor. Inhibition of TGF- $\beta-S m a d 2 / 3$ signaling with SD-208 significantly attenuated the upregulation of miRNA-181b. Knockdown of Smad4 in gastric cancer cells strongly attenuated the upregulation of miRNA-181b. Moreover, miR-181b was found to directly target the 3' untranslated region (3'UTR) of Timp3 mRNA affecting TGF$\beta$-induced EMT. Conclusions: Our results elucidate a novel mechanism through which the TGF- $\beta$ pathway regulates the EMT of gastric cancer cells by increasing the levels of miRNA-

Q. Zhou, X. Zheng and L. Chen contributed equally to this work.

Jingting Jiang, M.D.

and Changping Wu, M.D.

Department of Tumor Biological Treatment and Research Center for Cancer

Immunotherapy Technology of Jiangsu Province, the Third Affiliated Hospital, Soochow University, Jiangsu Changzhou 213003, (China)

Tel. +86 519-68870978, E-Mail jiangjingting@suda.edu.cn / wcpjjt@163.com
\end{abstract}

KARGER 
181b to target Timp3 via the Smad2/3/4-dependent pathway. These findings provide insights into the cellular and environmental factors regulating EMT, which may guide future studies on therapeutic strategies targeting these cells.

\section{Introduction}

Gastric cancer remains the fourth most commonly diagnosed cancer globally and the second most common cause of cancer mortality worldwide [1, 2]. Transforming growth factor $\beta$ (TGF- $\beta$ ) can act as a suppressor or promoter of cancer progression [3-5]. These ligands signal through the heteromeric complex of transmembrane serine/threonine kinases, the type I and type II receptors (TGFRI and TGFRII), and activate both the Smad family of transcription factors and non-Smad signaling pathways [6]. TGF- $\beta$ has a dual role in cancer: It limits proliferation in epithelial cells and early-stage cancer cells, whereas in late-stage cancer, it accelerates cancer progression and metastasis $[7,8]$. In the cancer niche, TGF- $\beta$ can be produced and secreted into the extracellular environment by both cancer cells and host cells such as lymphocytes, macrophages and dendritic cells [9-11].

Epithelial-to-mesenchymal transition (EMT) occurs in normal physiological processes essential for embryogenesis, tissue morphogenesis and wound healing but is also tightly linked to pathological conditions including fibrosis and cancer progression [12-19]. During EMT, epithelial cells typically lose their epithelial characteristics, including cell polarity and cell-cell contact, and acquire a spindle-shaped migrating phenotype. The key event in EMT is the switch of E-cadherin to $\mathrm{N}$-cadherin, which renders the single cell more motile and invasive [20-23]. TGF- $\beta$ is a major inducer of EMT [24, 25]. Several transcription factors including the zinc finger transcription factors Snail, Slug and Zeb play active roles guiding the EMT process. TGF- $\beta$-induced EMT has been suggested to be associated with development and progression of gastric cancer.

MicroRNAs (miRNAs) are small (20-30 nucleotides) noncoding RNAs that posttranscriptionally regulate gene expression through canonical base pairing between the miRNA seed sequence (nucleotides 2-8 at the $5^{\prime}$ end) and the complementary sequence in the 3' untranslated region (3'UTR) of the target mRNA [26-28]. The net effect of these events elicits either translational repression or degradation of targeted mRNAs. Recently, several studies have implicated aberrant miRNA expression in the development and metastatic progression of mammary tumors [29]. miRNA-181 family members are the most upregulated miRNAs in gastric cancer. miRNA-181 was initially identified as a hematopoietic lineage modulator. The significant roles of miR-181 in cancer were then revealed by various studies [30-32]. It has been reported that miR-181b was highly overexpressed in gastric tumors compared to normal gastric tissue and therefore this miRNA holds great potential as a prognostic biomarker in late stage gastric cancer. miRNA-181b was also associated with multi drug resistance by targeting BCL2. However, the mechanism underlying the role of TGF- $\beta$-induced miRNA-181b in the tumorigenesis and metastasis of gastric cancer has not been fully elucidated. Additionally, the direct target gene for miRNA-181b involved in TGF$\beta$-induced EMT needs to be identified.

Smads are recognized as downstream intracellular proteins in the signaling pathway of TGF- $\beta$, which is mediated through a heteromeric complex of two types of transmembrane receptors, TGF- $\beta$ receptor type I and II [6]. Smad4 is a central mediator of TGF- $\beta$ intracellular signaling [33]. However, the requirement of Smad4 in TGF- $\beta$-induced EMT and gastric cancer metastasis remains unclear. In this article, we report the contribution of miRNA-181b to TGF- $\beta$-induced EMT in vitro. By investigating the phenotypic changes and alterations in TGF- $\beta$ signaling responses following Smad4 knockdown using RNAi approaches in gastric cancer cells, we show a critical stimulatory function for Smad4 in TGF- $\beta$-driven gastric cancer progression. 


\section{Cellular Physiology Cell Physiol Biochem 2016;39:453-466 \begin{tabular}{ll|l} 
and Biochemistry $10.1159 / 000445638$ & $\begin{array}{l}\text { C) } 2016 \text { The Author(s). Published by S. Karger AG, Basel } \\
\text { Published online: July 07, } 2016\end{array}$ \\
www.karger.com/cpb
\end{tabular} Zhou et al.: miRNA-181b and Gastric Cancer Metastasis}

\section{Materials and Methods}

Cell culture and reagents

The human gastric cancer cell lines MKN-28, SGC-7901, HGC-27, AGS and HEK 293T were obtained from our institute. Cells were cultured in Dulbecco's modified Eagle's medium (Hyclone, Logan, UT, United States) supplemented with $10 \%$ heat-inactivated fetal bovine serum (FBS; Gibco, CA, United States). All cells were cultured at $37^{\circ} \mathrm{C}$ in a humidified incubator with $5 \% \mathrm{CO}_{2}$.

miRNA mimic and inhibitor

HGC-27 cells were transfected with miRIDIAN miRNA-181b mimic or mirCURY LNATM miRNA-181b inhibitor using Lipofectamine ${ }^{\mathrm{TM}} 2000$ according to the manufacturer's protocol. After $24-48 \mathrm{~h}$, cells were incubated with TGF- $\beta$ as stated above.

Wound healing assay

Gastric cell lines were cultured in six-well plates (seeding density $1 \times 10^{6}$ cells/well). Confluent cell monolayers were disrupted by standardized wound scratching using a sterile $200 \mu \mathrm{l}$ pipette tip and incubated in culture medium with $1 \%$ FBS without or with $5 \mathrm{ng} / \mathrm{ml} \mathrm{TGF}-\beta$ for $48 \mathrm{~h}$. Migration of cells into the bare area and recovery of the monolayer were monitored for $48 \mathrm{~h}$ by a phase contrast microscope and digitally photographed (Nikon Diaphot 300; Nikon, Tokyo, Japan).

Transwell invasion assay

Analyses of tumor cell migration and invasion were carried out using transwell chambers (Corning, United States). After $48 \mathrm{~h}$ transfection with miRNA-181b mimic or inhibitor, $2 \times 10^{5} \mathrm{HGC}-27$ or AGS cells in serum-free medium were collected and seeded in an upper chamber containing a non-coated $8 \mu \mathrm{m}$ membrane. Next, $500 \mu \mathrm{L}$ medium with $20 \%$ FBS were added to the lower chamber. For the invasion assay, chambers were coated with extracellular Matrigel (BD Biosciences, United States). Cells were cultured at $37^{\circ} \mathrm{C}$ in a humidified incubator with $5 \% \mathrm{CO}_{2}$. Non-migrating or non-invading cells in the upper chamber were removed with a cotton swab and cells that had migrated to or invaded the bottom chamber were fixed and stained with $0.1 \%$ crystal violet. Nine fields at $\times 100$ magnification were randomly selected and cell numbers counted. The results were averaged among three independent experiments.

\section{Real-time quantitative PCR}

Total RNA was extracted from gastric cancer cells (each experiment contained three repeats per group) by homogenization and lysis in TriPure reagent (Roche Applied Science, Mannheim, Germany) using a high-speed homogenizer. The concentration and purity of mRNA were evaluated using a Nano-Drop 2000 spectrophotometer (Thermo Fisher Scientific, MA, United States). Real-time quantitative PCR (qPCR) was performed following reverse transcription using a Roche LC 480 system with SYBR ${ }^{\circledR}$ Premix (TaKaRa, Inc., Dalian, China). Glyceraldehyde 3-phosphate dehydrogenase (GAPDH) was used as an internal control.

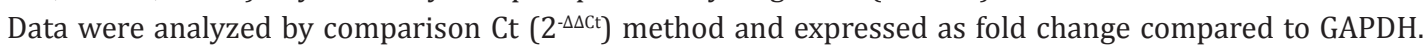
The relative level of mature miRNA-181b in gastric cells was calculated with respect to U6 RNA (internal control). Each sample was analyzed in triplicate.

\section{Immunofluorescence}

Cells were seeded on glass cover slips (Fisher, PA, United States) and cultured as previously described. The cells were fixed by incubation in 4\% paraformaldehyde for 5 min and permeabilized in PBS containing $0.1 \%$ Triton X-100 for $10 \mathrm{~min}$. Non-specific binding was blocked with 5\% BSA for $30 \mathrm{~min}$. F-actin was stained at a 1:1000 dilution in 2\% BSA of FITC-conjugated phalloidin (Sigma-Aldrich, St. Louis, MO, United States) for at least $30 \mathrm{~min}$. All washes were done in $1 \times$ PBS. An anti-fade solution containing DAPI (Vector Laboratories, CA, United States) was used in mounting the slides. Images were taken at room temperature with an Axiocam digital camera attached to a Zeiss microscope. Axiovision was used to acquire the image. Adobe Photoshop was used to merge images.

Western blot analysis

Cells were homogenized briefly in 10 volumes of lysis buffer containing (in mM) 20 Tris-HCl (pH 7.4), $150 \mathrm{NaCl}, 2.5$ EDTA, $50 \mathrm{NaF}, 0.1 \mathrm{Na}_{4} \mathrm{P}_{2} \mathrm{O}_{7}, 1 \mathrm{Na}_{3} \mathrm{VO}_{4}, 1 \mathrm{PMSF}, 1 \mathrm{DTT}, 0.02 \%$ (v/v) protease cocktail (SigmaAldrich), $1 \%(\mathrm{v} / \mathrm{v})$ Triton X-100 and 10\% (v/v) glycerol. The homogenates were centrifuged twice at KARGER 


\section{Cellular Physiology Cell Physiol Biochem 2016;39:453-466 \\ \begin{tabular}{ll|l} 
and Biochemistry $10.1159 / 000445638$ & $\begin{array}{l}\text { C) } 2016 \text { The Author(s). Published by S. Karger AG, Basel } \\
\text { Published online: July 07, } 2016\end{array}$
\end{tabular} \\ Zhou et al.: miRNA-181b and Gastric Cancer Metastasis}

$20,000 \times g$ at $4^{\circ} \mathrm{C}$ for $15 \mathrm{~min}$, and the supernatants were saved as total protein. Protein concentrations were determined by the BCA method. Equal amounts of proteins were separated by SDS-PAGE and transferred to a PVDF membrane (Bio-Rad, Hercules, CA, United States). Western blot analysis was performed under standard conditions with specific anti-Smad2/3 (Ab63672; Abcam, United States), anti-Smad4 (Ab40759, Abcam), anti-phospho Smad2/3 (Ab63399, Abcam) and anti-GAPDH (Ab8245, Abcam). The immunoreaction was visualized using an enhanced chemiluminescence detection kit (Amersham, London, UK), exposed to X-ray film, and quantified by densitometry with a video documentation system (Gel Doc 2000, Bio-Rad).

\section{Smad4 knockdown cell lines}

The target siRNAs against human Smad4 (GenBank, NM_005359.5) for RNAi were designed as follows: siSmad4, 5'-GCACAACACAACACAACACAACACAAGGTTGGTTGCTAAAA-3' and control RNAi sequence, 5'-TTCTCCCCGAACAACAACGTGTCACCACCACGT-3'. The lentiviral vector system was purchased from Clontech. This vector system includes three plasmids, the pLVX-U6-puro vector, psPAX and pMD2G. The targeting sequence was subcloned into the pLVX-U6-puro vector, which contained the U6 promoter and puromycin gene (puro). The resulting lentiviral vector containing Smad4 shRNA was named RNAi-Smad4, and its sequence was confirmed by PCR and sequencing. The lentiviral vector containing the negative control sequence for Smad4 shRNA (RNAi-NC) with a non-silencing sequence was used as the infection control for lentiviral vector production and cell infection. Lentiviral vectors were produced by transient transfection of HEK293 cells according to standard protocol as previously reported [22, 23]. The HEK293 cell line was supplemented with 10\% FBS and when subconfluent was transfected with $1800 \mu$ DNA solution: $10 \mu \mathrm{g}$ LV-shSmad4, $10 \mu \mathrm{g}$ psPAX and $10 \mu \mathrm{g}$ pMD2G. All virus stocks were produced by Lipofectamine-mediated transfection. After transfection for $48 \mathrm{~h}$, cell supernatants containing viral particles were filtered using a $0.45-\mu \mathrm{m}$ Steriflip vacuum filtration system (Millipore) and concentrated by ultracentrifugation at 25,000 $\mathrm{rpm}$ at $4^{\circ} \mathrm{C}$. The titer of the virus was tested according to the expression level of GFP. The day before infection, HGC-27 cells were seeded on dishes with a confluence of $30 \%-40 \%$. On the day of infection, the HGC27 cells were infected by packaged lentiviral particles. Parallel non-infected HGC-27 cells were observed simultaneously. Cells were cultured in normal growth medium for $72 \mathrm{~h}$ after infection. Transfection with RNAi-NC as infection control was performed as described previously. Cells were subsequently propagated in selection medium containing puromycin.

\section{Luciferase assay}

To confirm the targeting between miR-181b and the Timp3 messenger RNA 3'UTR, a luciferase assay was used. Part of the wild type 3'UTR (WT-3'UTR) and mutated 3'UTR (Mut-3'UTR) of Timp3 messenger RNA, containing the putative miR-181b or mutated miR-181b binding sites, were synthesized and inserted downstream from a luciferase reporter gene in a pGL3 plasmid (Promega, San Luis Obispo, CA, United States). HGC-27 cells were seeded in 24-well plates $\left(8 \times 10^{4}\right.$ cells per well $)$ and the next day transfected with $300 \mathrm{ng}$ of the WT or Mut 3'UTR luciferase reporter construct, together with a miR-181b or a scrambled precursor (final concentration $20 \mathrm{nM}$; Dharmacon, Lafayette, CO, United States). Each well was co-transfected with $50 \mathrm{ng}$ of the renilla plasmid pGL3 (Promega) as an internal transfection control. Cells were collected $48 \mathrm{~h}$ after transfection, and luciferase activity was measured with a dual-luciferase reporter assay (Promega). Luciferase activity was normalized to Renilla luciferase activity and expressed as the ratio between the luciferase activity of the WT-3'UTR plasmid and the Mut-3'UTR plasmid. Every luciferase assay experiment was repeated at least three times.

\section{Statistical analysis}

Data were expressed as means \pm SEM. Statistical significance was determined using ANOVA or repeated ANOVA for multiple comparisons or repeated measurements. Significant differences between two mean values were estimated using Student's t-test. A $P$ value $<0.05$ was considered statistically significant.

\section{Results}

Effect of TGF- $\beta$ on HGC-27 and AGS cell migration, invasion and EMT

EMT may contribute to the metastatic potential of tumor cells. Previous studies [1518] have demonstrated that TGF- $\beta$ can induce EMT in tumor cells. EMT is characterized by 


\section{Cellular Physiology Cell Physiol Biochem 2016;39:453-466 \begin{tabular}{ll|l} 
DOI: 10.1159/000445638 & $\begin{array}{l}\text { O 2016 The Author(s). Published by S. Karger AG, Basel } \\
\text { www.karger.com/cpb }\end{array}$
\end{tabular}

Fig. 1. Effect of TGF- $\beta$ on HGC-27 and AGS cell invasion, migration and EMT. (A) Transwell collagen assays showing the TGF- $\beta$ induced invasion of HGC27 and AGS cells toward $10 \%$ FBS. Membranes were fixed and evaluated for cell numbers. A representative picture of the membranes showing invading HGC-27 and AGS cells and quantification of the invasion assay is shown. (B) Wound healing assays showing the migration of TGF- $\beta$-treated HGC-27 and AGS cells. Quantification of wound closure capacity is shown. (C) qPCR results showing the expression of EMT markers (E-cadherin, Vimentin, Snail1 and Zeb1) in HGC-27 cells at baseline and after TGF- $\beta 1$ treatment (5 ng/ml, $48 \mathrm{~h}$ ). All values represent mean \pm SEM from three independent experiments. ${ }^{*} P$ $<0.05,{ }^{* *} P<0.01$ vs. the corresponding group.

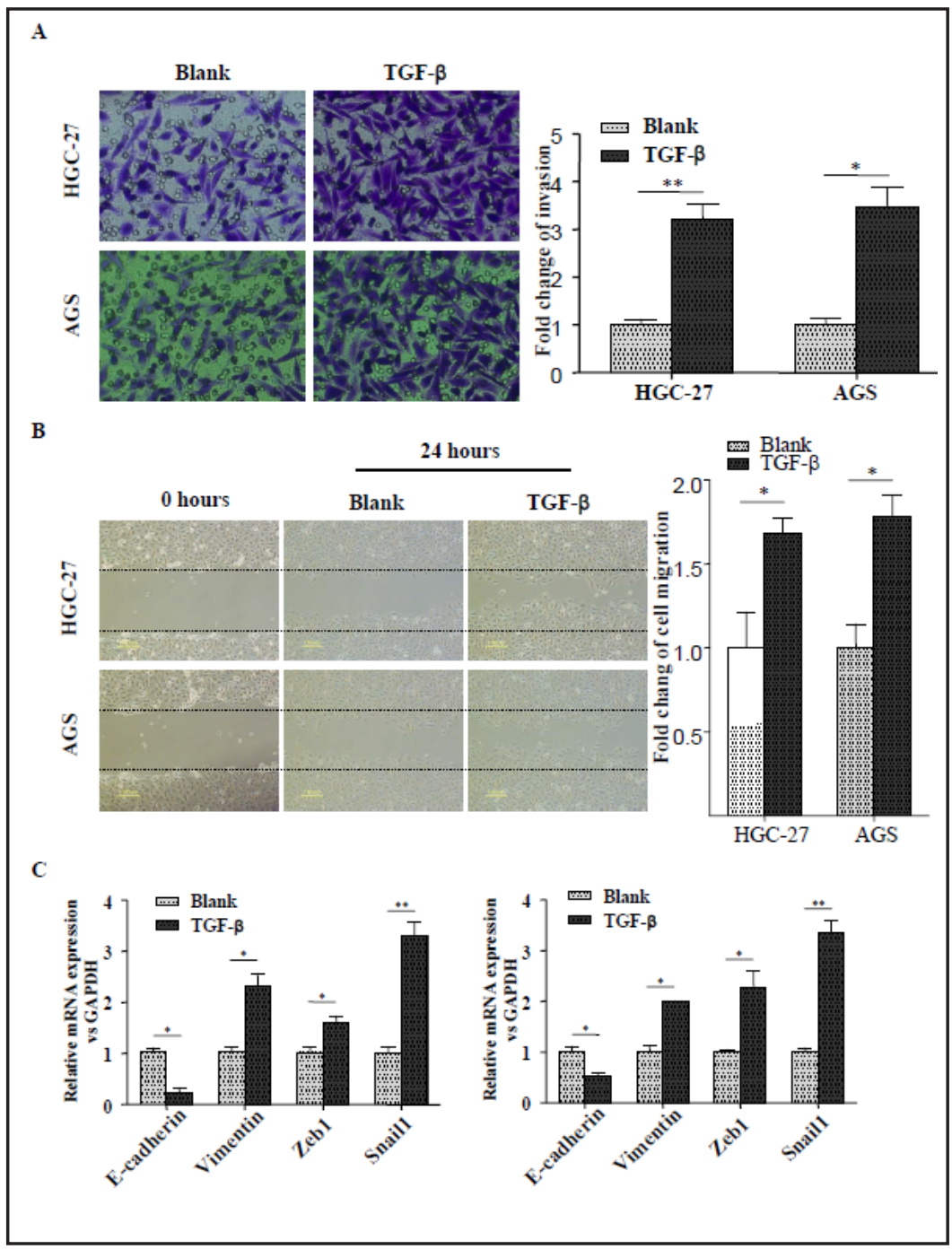

a clear switch to spindle shape morphology, accompanied by downregulation of E-cadherin and upregulation of Vimentin, Zeb1 and Snail1. HGC-27 and AGS cell lines were selected that maintain a stable epithelial morphology in the absence of TGF- $\beta$. Treatment of these two cell lines with TGF- $\beta$ ( $5 \mathrm{ng} / \mathrm{ml})$ for $48 \mathrm{~h}$ resulted in a strong enhancement of cell migration and invasion by transwell invasion assays (Fig. 1A) and wound healing, respectively (Fig. 1B). Consistently, qPCR analysis showed the induction of Vimentin, Zeb1 and Snail1 and a reduction in E-cadherin mRNA expression (Fig. 1C).

Expression of miRNA-181b induced by TGF- $\beta$ in gastric cancer cells

Recently, several studies $[27,28,30,34,35]$ have implicated aberrant miRNA expression in the development and metastatic progression of mammary tumors. These studies reported that miR-181 expression was dramatically and selectively upregulated in metastatic breast tumors, particularly triple-negative breast cancers, and was highly predictive for decreased overall survival in human breast cancer patients. We therefore focused on miR-181 family members in our study for their potential function in gastric cancer as downstream effectors of TGF- $\beta$ signaling. As shown in Fig. 2, gastric cancer cells (MKN28, SGC-7901, AGS and HGC-27) treated with TGF- $\beta$ showed increasing expression of pre-miRNA-181b and mature miRNA-181b by qPCR analysis. 
Fig. 2. Expression of miRNA-181b induced by TGF- $\beta$ in gastric cancer cells. qPCR results show the expression of precursor and mature miRNA-181b in MKN-28, SGC-7901, AGS and HGC-27 gastric cell lines at baseline and after TGF- $\beta 1$ treatment (5 ng/ml; 4, 12, 24 and $48 \mathrm{~h}$ ). All values represent mean \pm SEM from three independent experiments. ${ }^{*} P$ $<0.05,{ }^{* *} P<0.01,{ }^{* * *} P<0.001$ vs. the corresponding group.

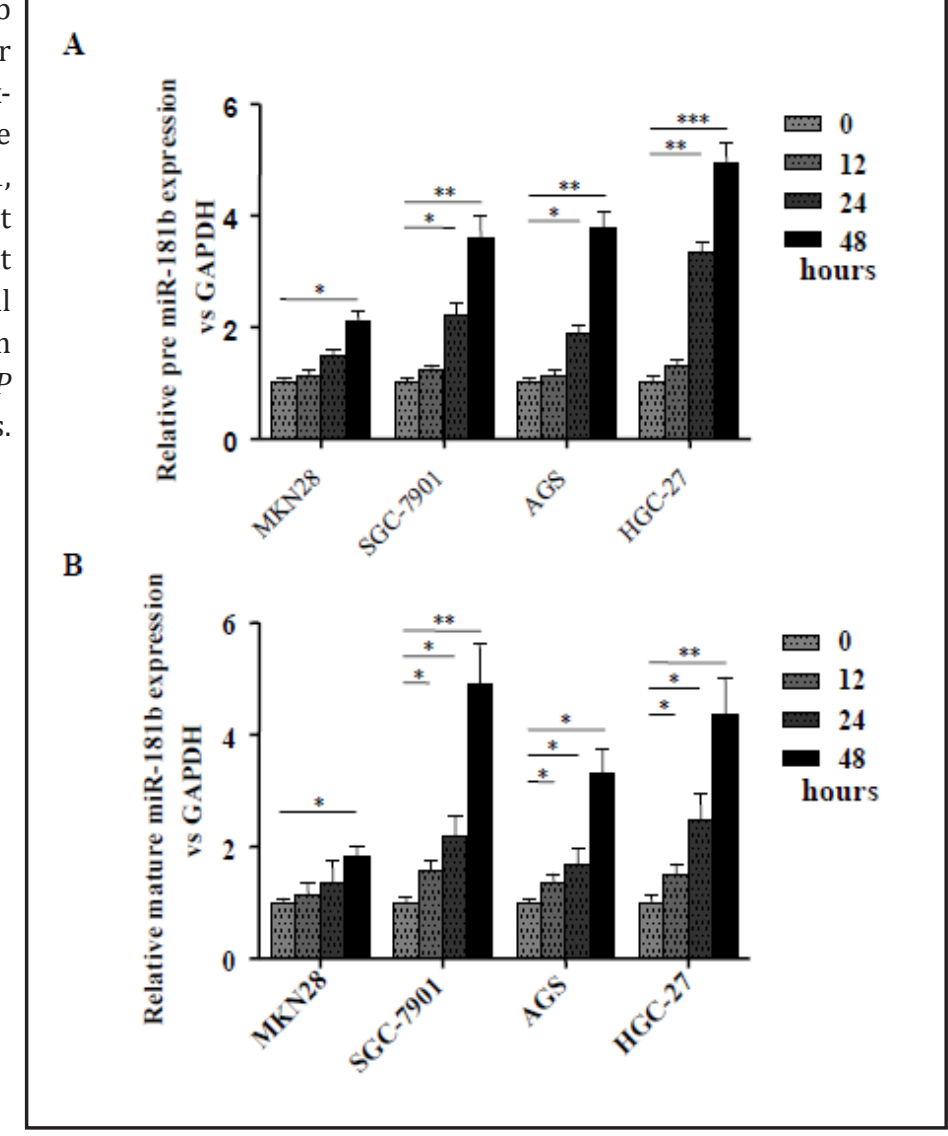

A

miRNA-181b is involved in TGF- $\beta$-mediated migration, invasion and EMT

To study the cellular function of miRNA-181b in gastric cancer, we transfected miRNA181b mimic, inhibitor or siRNA duplexes with non-specific sequences as NC into HGC-27 cells with or without TGF- $\beta$ treatment. miRNA-181b levels were effectively elevated by transfection of the mimic and decreased by transfection of the inhibitor (data not shown). Inhibition of miRNA-181b significantly suppressed TGF- $\beta$-induced HGC-27 cell invasion compared with control cells $(P<0.05$, Fig. 3A), but had no effect on normal HGC-27 cells. However, the in vitro scratch wound-healing assay showed that miRNA-181b inhibition significantly suppressed both TGF- $\beta$-induced and normal HGC-27 cell migration $(P<0.05$, Fig. 3B). Interestingly, the over-expression of miRNA-181b only promoted the migration and invasion of normal but not TGF- $\beta$-induced HGC-27 cells. Consistently, F-actin staining showed changes in the morphology of HGC-27 cells (Fig. 3C) and qPCR analysis (Fig. 3D) showed that miRNA-181b inhibition significantly suppressed the EMT of TGF- $\beta$-induced HGC-27 cells, evaluated by mRNA expression of Vimentin, Zeb1, Snail1 and E-cadherin $(P$ $<0.05$, Fig. 3D). These results suggested that miRNA-181b is a regulator of TGF- $\beta$-induced migration, invasion and EMT in HGC-27 cells.

\section{Inactivation of TGFRII attenuates TGF- $\beta$-induced miRNA-181b expression}

TGF- $\beta$ superfamily ligands bind to a type II receptor, which recruits and phosphorylates a type I receptor. The type I receptor then phosphorylates receptor-regulated Smads (such as Smad2) which can now bind the common-mediator (co)-Smad Smad4. In order to investigate the role of the Smad2/3 pathway in the upregulation of miRNA-181b in response to TGF- $\beta$, HGC-27 cells were treated with SD-208, a TGFRII inhibitor, which blocked TGF- $\beta$ signaling as determined by suppression of Smad 2 phosphorylation (Fig. 4A). Furthermore, treatment with SD-208 also blocked TGF- $\beta$-induced expression of pre-miRNA-181b (Fig. 4B) and mature miRNA-181b (Fig. 4C) as demonstrated by qPCR analysis. These results suggested 
A
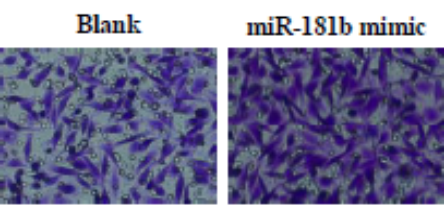

TGF- $\beta$

TGF- $\beta$
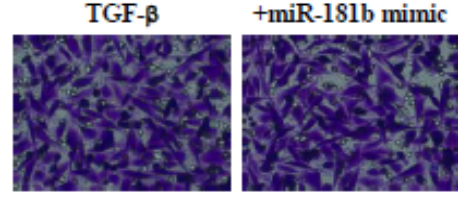

TGF- $\beta$
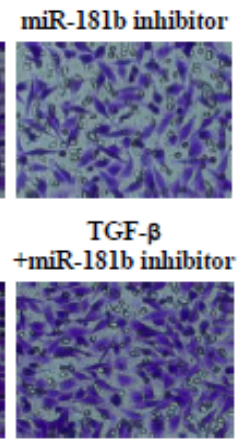

B

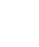

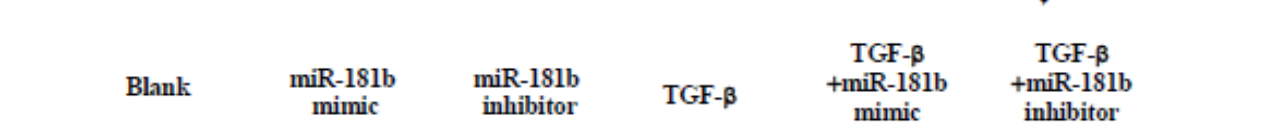

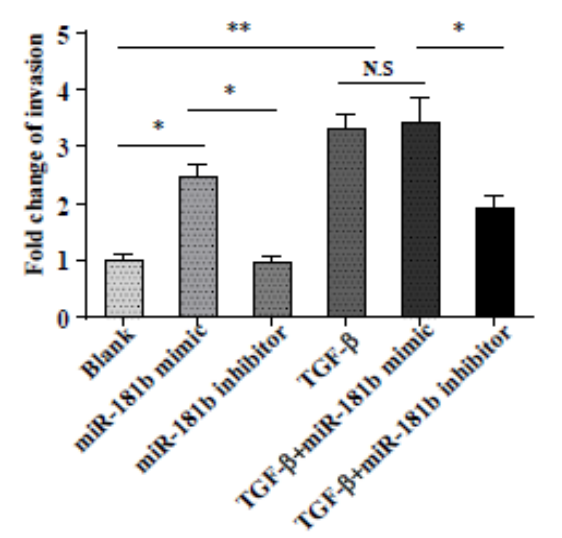

miR-181
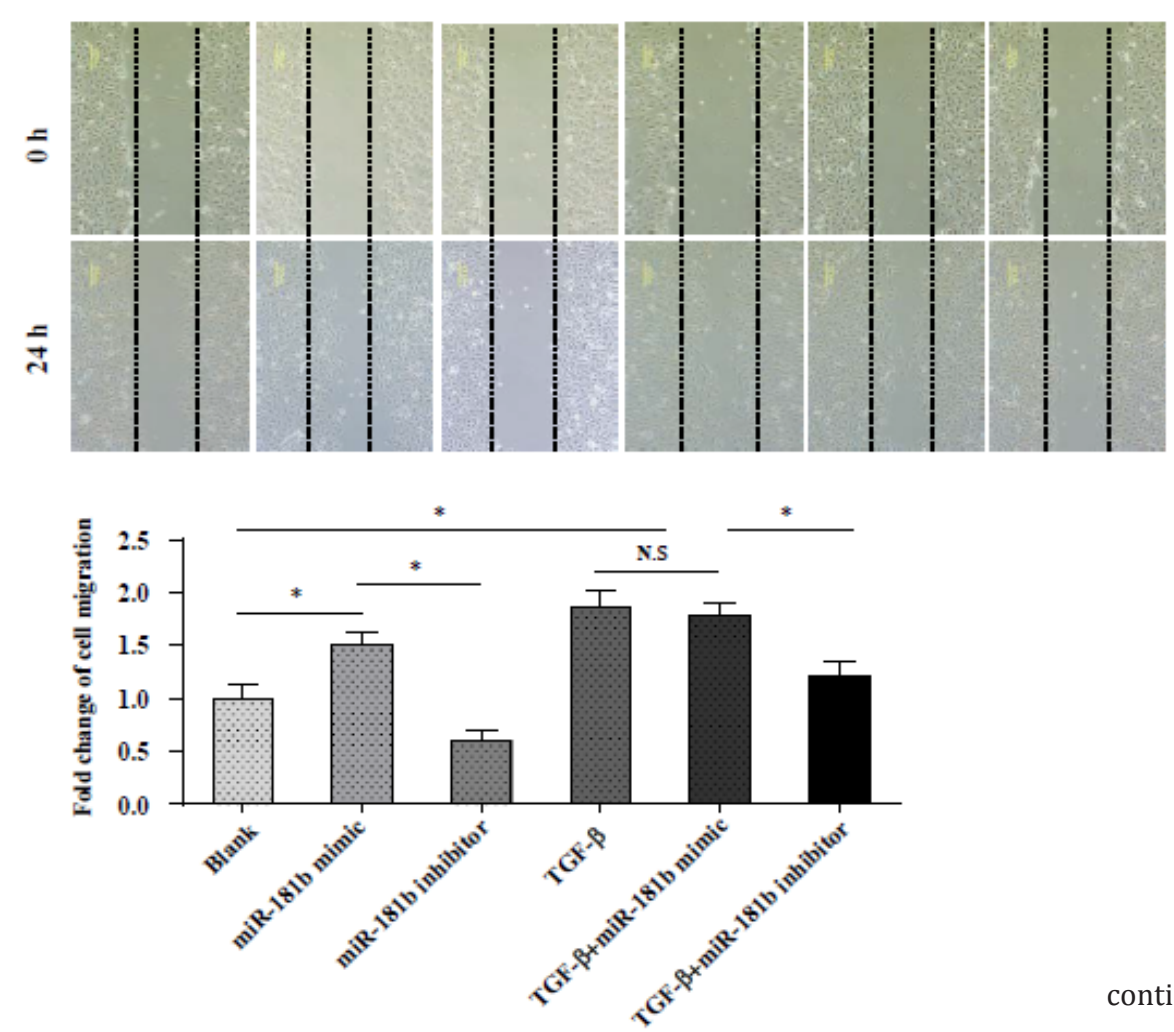

continued

Fig. 3. miRNA-181b inhibits TGF- $\beta$-mediated migration, invasion and EMT. (A) Transwell collagen assays showing the reduced invasion of HGC-27 cells toward 10\% FBS following the addition of miRNA-181b mimic or inhibitor during TGF- $\beta$ induction. Membranes were fixed and evaluated for cell numbers. A representative picture of the membranes showing invading HGC-27 cells and quantification of the invasion assay is shown. (B) Wound healing assays showing reduced migration of HGC-27 cells following the addition of miRNA-181b mimic or inhibitor during TGF- $\beta$ induction. Quantification of wound closure capacity is shown. (C) F-actin staining shows changes in the morphology of HGC-27 cells. Green staining, F-actin; blue staining, DAPI. (D) qPCR results showing the expression of EMT markers (E-cadherin, Vimentin, Snail1 and Zeb1) in HGC-27 cells at baseline and after TGF- $\beta 1$ treatment ( $5 \mathrm{ng} / \mathrm{ml}, 48 \mathrm{~h}$ ) following the addition of miRNA-181b mimic or inhibitor. All values represent mean \pm SEM from three independent experiments. ${ }^{*} P<0.05,{ }^{* *} P<$ 0.01 vs. the corresponding group. N.S, non-significant.

\section{KARGER}


C
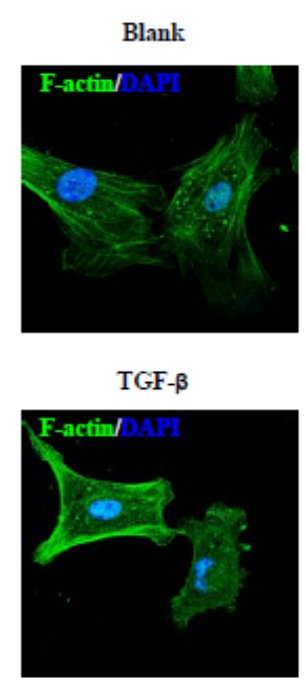

miR-181b mimic

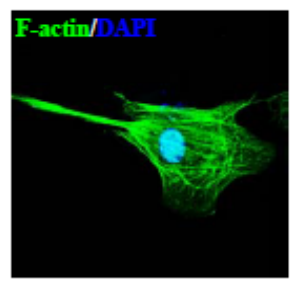

TGF- $\beta$ + miR-181b mimic

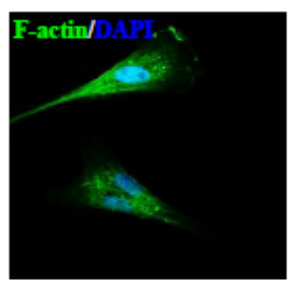

miR-181b inhibitor

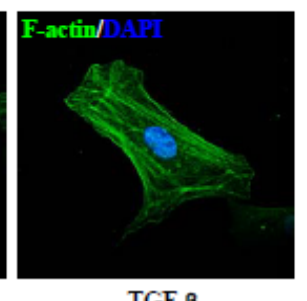

+miR-181b inhibitor

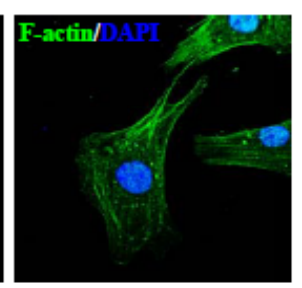

$\mathbf{D}$

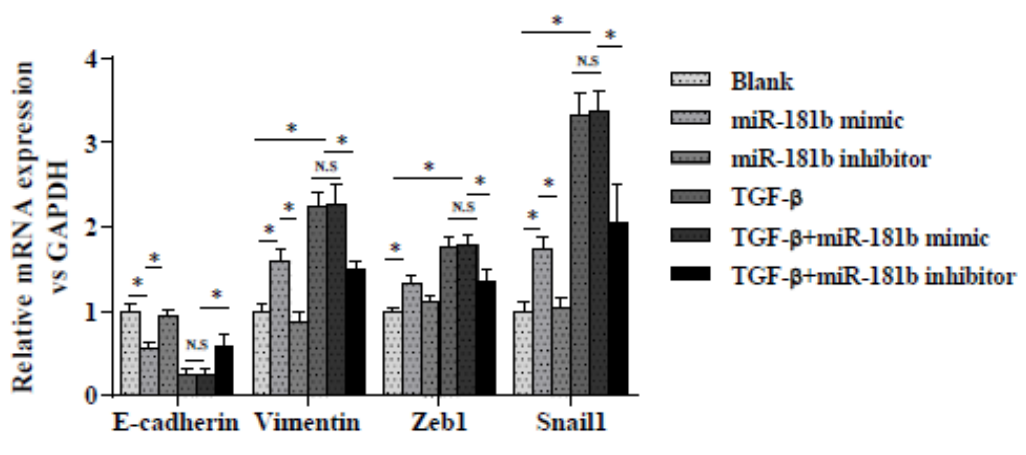

that the upregulation of miRNA-181b in response to TGF- $\beta$ requires the Smad2/3 signaling pathway

Smad4 is required for TGF- $\beta$-induced miRNA-181b expression in the early stage of TGF- $\beta$ induction

Receptor-activated Smads (Smad2 and Smad3 for TGF- $\beta$ ) are phosphorylated in SXSmotives in their C-terminals, where after they form complexes with the co-Smad Smad4. In order to investigate the contribution of $\mathrm{Smad} 2 / 3 / 4$ complexes in TGF- $\beta$-induced upregulation of miRNA-181b, we created a lentivirus-mediated stable Smad4 knockdown HGC-27 cell line, in which the extent of Smad4 knockdown was $>75 \%$ (Fig. 5A), while the level of phosphoSmad2 remained unchanged as shown by western blot analysis (Fig. 5B). Furthermore, Smad4 knockdown blocked TGF- $\beta$-induced expression of pre-miRNA-181b (Fig. 5C) from 4-24 h, and still showed a weak effect at $48 \mathrm{~h}$ as demonstrated by qPCR analysis. For the mature miRNA-181b transcript (Fig. 5D), Smad4 knockdown blocked expression from 4-12 $\mathrm{h}$ but only partially blocked expression from $24-48 \mathrm{~h}$. These results suggested that the upregulation of miRNA-181b in response to TGF- $\beta$ partially required the activation of the type I receptor of TGF- $\beta$ and the formation of Smad2/3/4 complexes.

Timp3 is a direct and functional target of miR-181b in gastric cancer cells

To explore the potential target of miR-181b involved in TGF- $\beta$ induction, we investigated whether miR-181b could regulate several reported targets (ATM, Bcl2, CREB1, CYLD and Timp3) in HGC-27 gastric cancer cells. We transfected miRNA-181b mimic, inhibitor or 
Fig. 4. Inactivation of TGFRII attenuates TGF- $\beta$-induced miRNA-181b expression. (A) Western blots showing inhibition of TGF$\beta$-induced Smad2 phosphorylation by SD-208 (1 $\mu \mathrm{M})$ in HGC-27 cells. Representative blots are shown $(\mathrm{n}=3)$. (B, C) qPCR results showing the inhibition of TGF- $\beta$-induced precursor and mature miRNA-181b expression by SD-208 (1 $\mu \mathrm{M})$ in HGC-27 cells (TGF- $\beta 5 \mathrm{ng} / \mathrm{ml} ; 4$, 12, 24 and $48 \mathrm{~h}$ ). All values represent mean \pm SEM from three independent experiments. ${ }^{*} P<$ $0.05,{ }^{* *} P<0.01$ vs. the corresponding group.
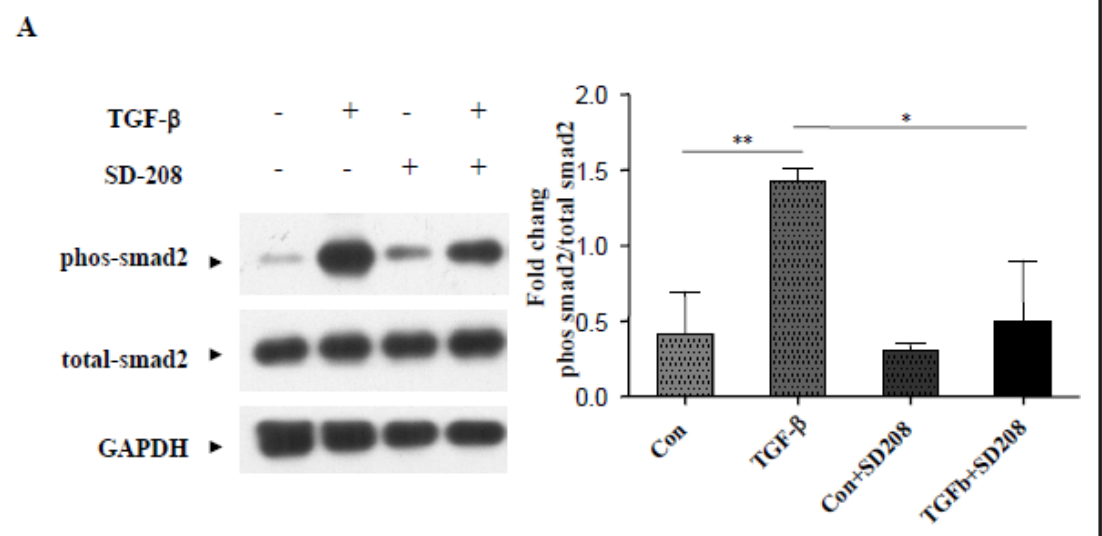

B

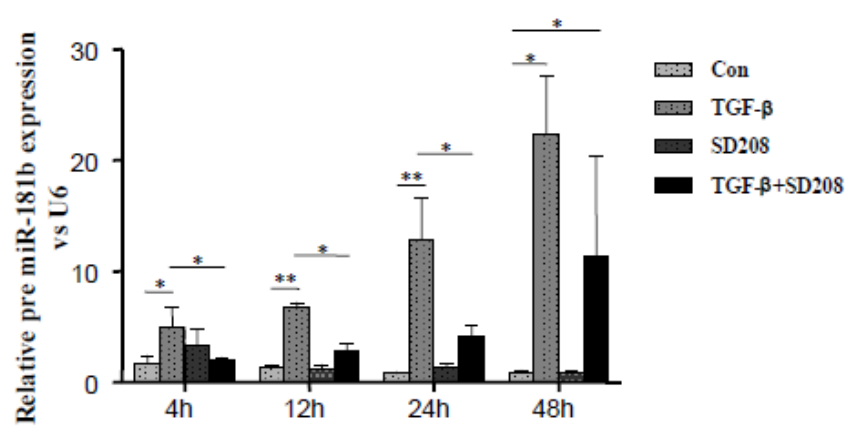

C

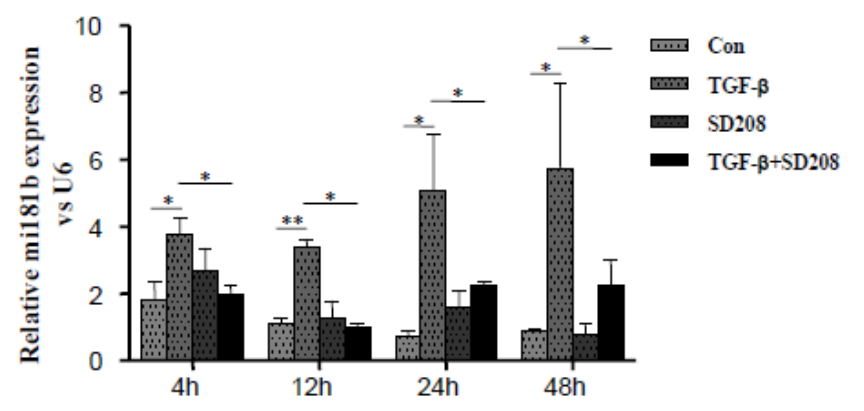

siRNA duplexes with non-specific sequences as NC into HGC-27 cells with or without TGF- $\beta$ treatment. Only Timp3 expression was regulated by miR-181b. Compared with the corresponding control, the level of Timp3 expression was significantly suppressed by miR181b mimic and was further suppressed with TGF- $\beta$ treatment (Fig. 6A). Moreover, Timp3 expression was upregulated by miRNA-181b inhibitor with or without TGF- $\beta$ (Fig. 6A).

We further performed luciferase reporter assays to determine whether miR-181b could directly target the 3'UTR of Timp3 in GC cells. The target sequence in the Timp3 WT-3'UTR or Mut-3'UTR was cloned into a luciferase reporter vector (Fig. 6B and C). HGC-27 cells were then transfected with the WT or Mut 3'UTR vector and miRNA-181b mimic. The results showed a significant decrease in luciferase activity when compared with miR-NC (Fig. 6B). In contrast, the activity of the Mut 3 'UTR vector was unaffected by simultaneous transfection with miR-181b mimic. The results suggested that Timp3 was a direct target of miR-181b in GC cells.

To examine the functional relationship between miR-181b and Timp3, we analyzed whether Timp3 can counteract the biological effect of TGF- $\beta$-induced miR-181b. We evaluated whether ectopic expression of Timp3 could rescue the suppressive effect of miR- 
A

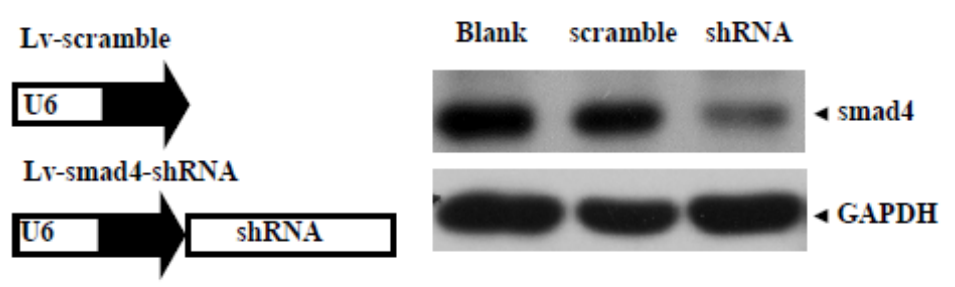

B
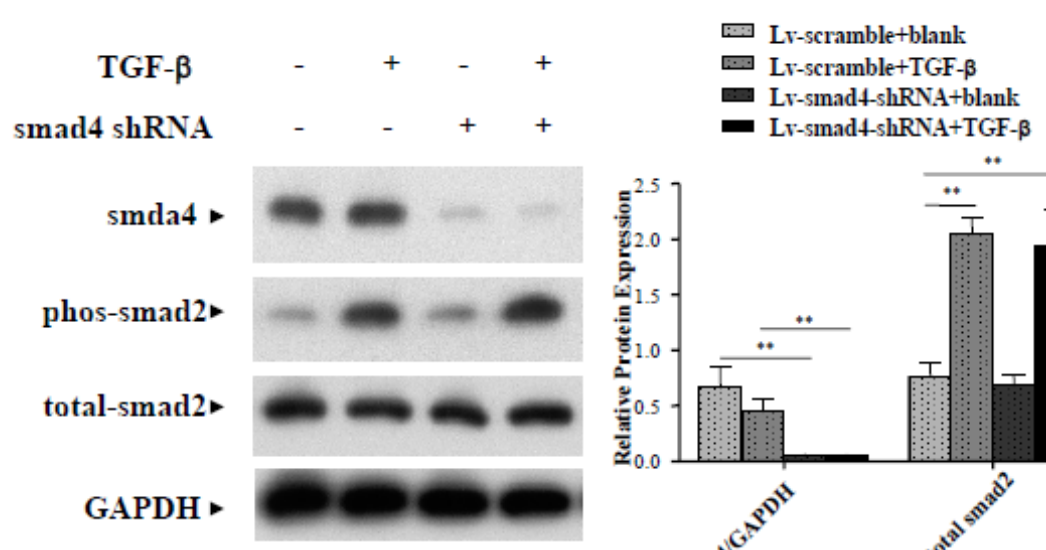

C

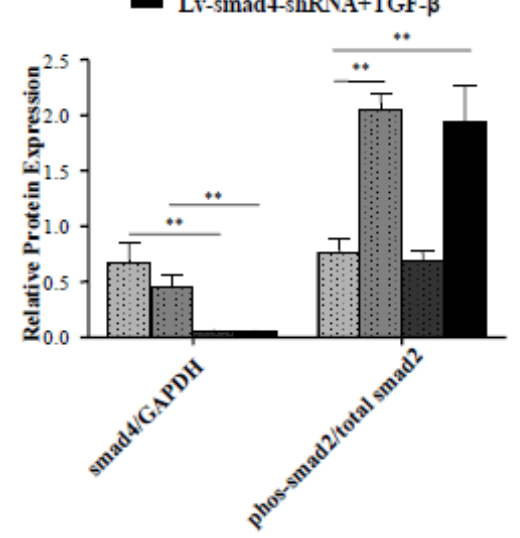
Lv-scrambletblank
Lv-scramble+TGF- $\beta$
- Lv-smad shRNA+blank
- Lv-smadt shRNA+TGF- $\beta$

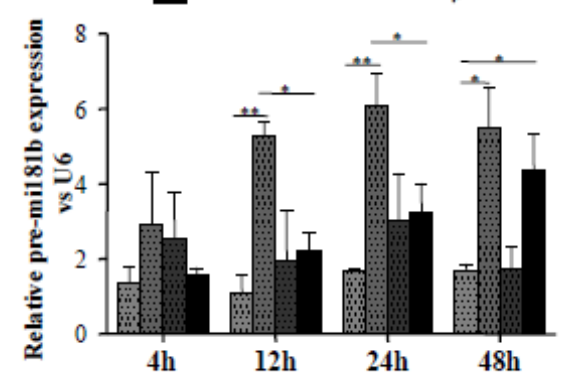

D

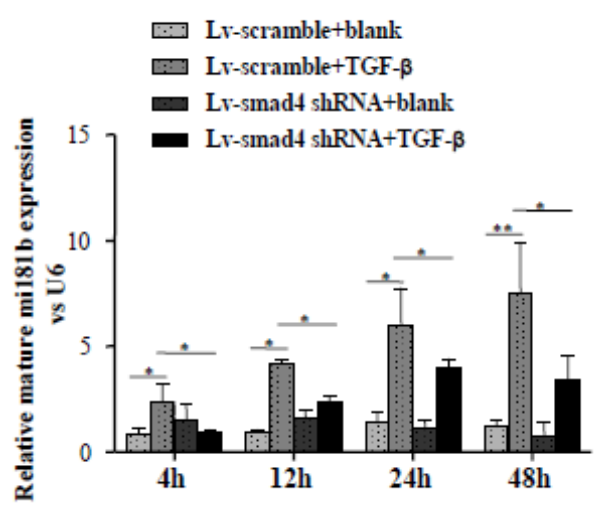

Fig. 5. Smad4 is required for TGF- $\beta$-induced miRNA-181b expression in the early stage of TGF- $\beta$ induction. (A) Schematic representative of lentiviruses encoding Smad4 shRNAs and immunoblotting analysis of Smad4 protein levels. (B) Western blots showing that Smad4 knockdown had no effect on TGF- $\beta$-induced Smad2 phosphorylation in HGC-27 cells. Representative blots are shown ( $\mathrm{n}=3$ ). (C, D) qPCR results showing the inhibition of TGF- $\beta$-induced precursor and mature miRNA-181b expression in HGC-27 cells (TGF- $\beta$ $5 \mathrm{ng} / \mathrm{ml} ; 4,12,24$ and $48 \mathrm{~h}$ ) after knockdown of Smad4. All values represent mean \pm SEM from three independent experiments. ${ }^{*} P<0.05,{ }^{* *} P<0.01$ vs. the corresponding group.

181b on TGF- $\beta$-induced EMT marker expression. HGC-27 cells were transfected for $48 \mathrm{~h}$ with pcDNA-Timp3, which encoded the full-length coding sequence without the 3'UTR region, followed by TGF- $\beta$ treatment, Results showed that ectopic expression of Timp3 partially rescued TGF- $\beta$-induced EMT marker expression (Fig. 6C). 
A

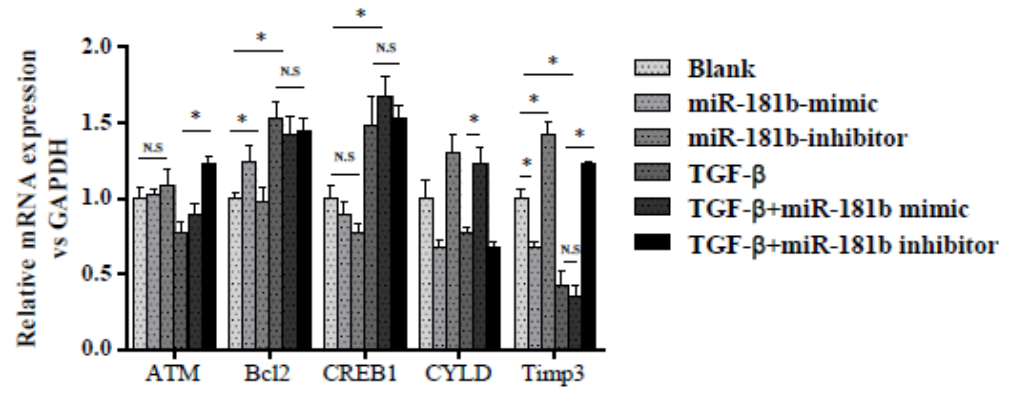

B

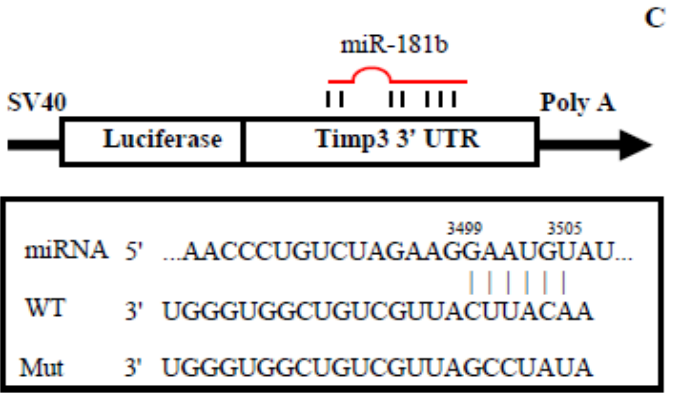

$\mathrm{C}$

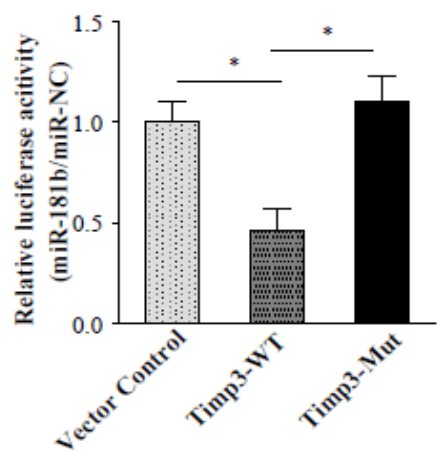

D

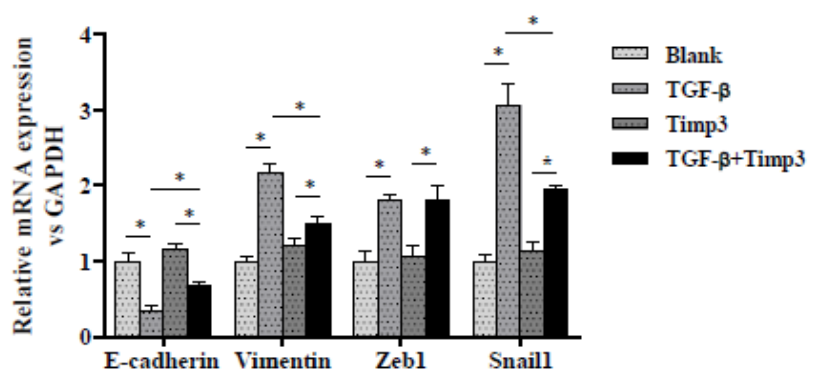

Fig. 6. Timp 3 is a direct and functional target of miR-181b in gastric cancer cells. (A) qPCR results showing the expression of reported targets ATM, Bcl2, CREB1, CYLD and Timp3 in HGC-27 cells at baseline and after TGF- $\beta 1$ treatment ( $5 \mathrm{ng} / \mathrm{ml}, 48 \mathrm{~h}$ ) following the infection of pcDNA-Timp3. (B) Schematic description of the hypothesized interactions between miR-181b and the wild-type (WT) or mutant (Mut) putative target regions in the 3'UTR of Timp3. (C) HGC-27 cells were transfected with vectors containing WT or Mut miRNA-181b target sites from the Timp3 3'UTR, or with empty vector (Ctrl), plus miRNA-181b or miR-NC. Luciferase activity was normalized to Renilla and presented as relative to miR-NC. ${ }^{*} \mathrm{P}<0.05$. (D) qPCR results showing the expression of EMT markers (E-cadherin, Vimentin, Snail1 and Zeb1) in HGC-27 cells at baseline and after TGF- $\beta 1$ treatment $(5 \mathrm{ng} / \mathrm{ml}, 48 \mathrm{~h})$ following the infection of pcDNA-Timp3. ${ }^{*} P<0.05,{ }^{* *} P<0.01 \mathrm{vs}$. the corresponding group. N.S, non-significant.

\section{Discussion}

Recent studies have demonstrated that miRNAs are key players in regulating tumor progression and metastasis. It is well established that miRNAs can function as tumor suppressors (e.g., miRNA-33a) and tumor promoters (e.g., miRNA-182) [34-36]. The clinical significance of miR-181 was also investigated in gastric cancer patients undergoing S-1 treatment. The efficacy of S-1 in the treatment of patients with advanced gastric cancer and patient survival were inversely correlated with the expression of miR-181 [37]. Thus, miR$181 \mathrm{~b}$ exhibits great potential as a prognostic biomarker in late-stage gastric cancer. More 


\section{Cellular Physiology Cell Physiol Biochem 2016;39:453-466 \begin{tabular}{l|l} 
DOI: 10.1159/000445638 & $\begin{array}{l}\text { O 2016 The Author(s). Published by S. Karger AG, Basel } \\
\text { www.karger.com/cpb }\end{array}$
\end{tabular} \\ Zhou et al.: miRNA-181b and Gastric Cancer Metastasis}

recently, the miR-181 family has been implicated in regulating specific steps in the TGF- $\beta$ induced metastatic cascade. Because metastasis is the major cause of death for gastric cancer patients, it stands to reason that defining the molecular mechanisms whereby miRNAs have an impact on metastasis may provide novel opportunities to treat metastatic gastric cancers. The miR-181 family contains four members (i.e., miRNAs 181a-d), all of which house identical seed sequences, suggesting that this miRNA family may exhibit redundancy in targeting mRNAs such as ATM, Bcl2, Creb1, CYLD and Timp3 [32, 38-40]. These targets regulated by miR-181b suggest that the function of miR-181b may be unique, depending on the type of tumor and cellular context. However, the exact mechanism through which the signaling pathway controls the expression of miRNA-181 family members remains to be determined.

Two distinct mechanisms have been reported in the regulation of TGF- $\beta$-induced miRNA expression. The downstream effector Smads were reported to bind and activate the promoter of miRNA-155 [41,42]. However, in the regulation of miR-21, Smad2/3 binds to the primary transcript of miR-21 by interacting with the Drosha processing complex to facilitate the maturation of miR-21 [43]. In the present study, in order to investigate which mechanism is involved in the regulation of TGF- $\beta$-induced miRNA-181b expression, we examined the contribution of Smad2/3 phosphorylation via the selective inhibition of TGFRII by SD-208. The TGF- $\beta$-induced precursor and mature miRNA-181b were partially inhibited after the treatment with SD-208, suggesting that regulation occurs at the transcriptional level. In the Smad2/3 pathway, to activate the promoter of miRNA-181b, Smad4 is essential for the formation of the Smad2/3/4 complex and translocation into the nucleus following TGF- $\beta$ stimulation $[44,45]$. Smad4 knockdown using lentivirus-mediated shRNA partially blocked TGF- $\beta$-induced precursor and mature miRNA-181b, consistent with results following treatment with SD-208, suggesting that the TGF- $\beta$-Smad2/3/4 pathway contributes to the regulation of TGF- $\beta$-induced miRNA-181b at the transcriptional level.

As there is usually an inverse relationship between the expression of miRNA and their targets, the identification of Timp3 as a target of miR-181b is of considerable interest. Timp3, an inhibitor of metalloprotease, induces apoptosis and inhibits angiogenesis, cell migration and invasion [46].

In conclusion, this study shows the contribution of the TGF- $\beta$-Smad $2 / 3 / 4$ pathway in the regulation of TGF- $\beta$-induced miRNA-181b at the transcriptional level and offers a molecular mechanism for the potential role of TGF- $\beta$-induced miR-181b in EMT through decreased expression of Timp3.

\section{Acknowledgments}

This work was supported by grants from the National Natural Science Foundation of China (Grant Nos. 31570877, 31570908, 81301960 and 31428005), the International Cooperation Project from Science and Technology Bureau of Changzhou (CZ20130020), the Program of Jiangsu Engineering Research Center for Tumor Immunotherapy (BM2014404), the Key R\&D Project of Science and Technology Department of Jiangsu Province (BE2015633), the Jiangsu Key Laboratory of Medical Science and Laboratory Medicine (JSKLM-2014-003) and the High-level Talents Training Project of Changzhou Health Bureau.

\section{Disclosure Statement}

The authors declare that there are no conflicts of interest.

\section{References}

1 Zhao CM, Hayakawa Y, Kodama Y, Muthupalani S, Westphalen CB, Andersen GT, Flatberg A, Johannessen H, Friedman RA, Renz BW, Sandvik AK, Beisvag V, Tomita H, Hara A, Quante M, Li Z, Gershon MD, Kaneko K, Fox JG, Wang TC, Chen D: Denervation suppresses gastric tumorigenesis. Sci Transl Med 2014;6:250ra115. 


\section{Cellular Physiology Cell Physiol Biochem 2016;39:453-466 \begin{tabular}{l|l|l|}
\hline DOI: 10.1159/000445638 & $\begin{array}{l}\text { C) 2016 The Author(s). Published by S. Karger AG, Basel } \\
\text { www.karger.com/cpb }\end{array}$
\end{tabular} \\ Zhou et al.: miRNA-181b and Gastric Cancer Metastasis}

2 Carcas LP: Gastric cancer review. J Carcinog 2014;13:14.

3 Rafehi S, Ramos-Valdes Y, Bertrand M, McGee J, Prefontaine M, Sugimoto A, DiMattia G, Shepherd TG: TGFbeta signalling regulates epithelial-mesenchymal plasticity in ovarian cancer ascites-derived spheroids. Endocr Relat Cancer DOI:10.1530/ERC-15-0383.

4 Chen C, Zhao KN, Masci PP, Lakhani SR, Antonsson A, Simpson PT, Vitetta L: TGFbeta isoforms and receptors mRNA expression in breast tumours: prognostic value and clinical implications. BMC Cancer 2015;15:1010.

5 Ikushima H, Miyazono K: TGFbeta signalling: a complex web in cancer progression. Nat Rev Cancer 2010;10:415-424.

6 Derynck R, Zhang YE: Smad-dependent and Smad-independent pathways in TGF-beta family signalling. Nature 2003;425:577-584.

7 Au HK, Chang JH, Wu YC, Kuo YC, Chen YH, Lee WC, Chang TS, Lan PC, Kuo HC, Lee KL, Lee MT, Tzeng CR, Huang YH: TGF-betaI Regulates Cell Migration through Pluripotent Transcription Factor OCT4 in Endometriosis. PLoS One 2015;10:e0145256.

8 Muthusamy BP, Budi EH, Katsuno Y, Lee MK, Smith SM, Mirza AM, Akhurst RJ, Derynck R: ShcA Protects against Epithelial-Mesenchymal Transition through Compartmentalized Inhibition of TGF-beta-Induced Smad Activation. PLoS Biol 2015;13:e1002325.

9 Massague J: TGFbeta signalling in context. Nat Rev Mol Cell Biol 2012;13:616-630.

10 Feng XH, Derynck R: Specificity and versatility in tgf-beta signaling through Smads. Annu Rev Cell Dev Biol 2005;21:659-693.

11 Shi Y, Massague J: Mechanisms of TGF-beta signaling from cell membrane to the nucleus. Cell 2003;113:685-700.

12 Hu Q, Tong S, Zhao X, Ding W, Gou Y, Xu K, Sun C, Xia G: Periostin Mediates TGF-beta-Induced Epithelial Mesenchymal Transition in Prostate Cancer Cells. Cell Physiol Biochem 2015;36:799-809.

13 Ying X, Sun Y, He P: Bone Morphogenetic Protein-7 Inhibits EMT-Associated Genes in Breast Cancer. Cell Physiol Biochem 2015;37:1271-1278.

14 Scheel C, Weinberg RA: Cancer stem cells and epithelial-mesenchymal transition: concepts and molecular links. Semin Cancer Biol 2012;22:396-403.

15 Katsuno Y, Lamouille S, Derynck R: TGF-beta signaling and epithelial-mesenchymal transition in cancer progression. Curr Opin Oncol 2013;25:76-84.

16 Lamouille S, Derynck R: Cell size and invasion in TGF-beta-induced epithelial to mesenchymal transition is regulated by activation of the mTOR pathway. J Cell Biol 2007;178:437-451.

17 Miyazono K: Transforming growth factor-beta signaling in epithelial-mesenchymal transition and progression of cancer. Proc Jpn Acad Ser B Phys Biol Sci 2009;85:314-323.

18 Wendt MK, Allington TM, Schiemann WP: Mechanisms of the epithelial-mesenchymal transition by TGFbeta. Future Oncol 2009;5:1145-1168.

19 Bu F, Liu X, Li J, Chen S, Tong X, Ma C, Mao H, Pan F, Li X, Chen B, Xu L, Li E, Kou G, Han J, Guo S, Zhao J, Guo Y: TGF-beta1 induces epigenetic silence of TIP30 to promote tumor metastasis in esophageal carcinoma. Oncotarget 2015;6:2120-2133.

20 Kang X, Kong F, Wu X, Ren Y, Wu S, Wu K, Jiang Z, Zhang W: High glucose promotes tumor invasion and increases metastasis-associated protein expression in human lung epithelial cells by upregulating heme oxygenase-1 via reactive oxygen species or the TGF-beta1/PI3K/Akt signaling pathway. Cell Physiol Biochem 2015;35:1008-1022.

21 Shen Y, Wei Y, Wang Z, Jing Y, He H, Yuan J, Li R, Zhao Q Wei L, Yang T, Lu J: TGF-beta regulates hepatocellular carcinoma progression by inducing Treg cell polarization. Cell Physiol Biochem 2015;35:1623-1632.

22 Kalluri R, Weinberg RA: The basics of epithelial-mesenchymal transition. J Clin Invest 2009;119:14201428.

23 Gonzalez DM, Medici D: Signaling mechanisms of the epithelial-mesenchymal transition. Sci Signal 2014;7:re8.

24 Teng Y, Zhao L, Zhang Y, Chen W, Li X: Id-1, a protein repressed by miR-29b, facilitates the TGFbeta1induced epithelial-mesenchymal transition in human ovarian cancer cells. Cell Physiol Biochem 2014;33:717-730. 


\section{Cellular Physiology Cell Physiol Biochem 2016;39:453-466 \begin{tabular}{l|l|l} 
and BOI: 10.1159/000445638 & $\begin{array}{l}\text { ( ) 2016 The Author(s). Published by S. Karger AG, Basel } \\
\text { www.karger.com/cpb }\end{array}$
\end{tabular} \\ Zhou et al.: miRNA-181b and Gastric Cancer Metastasis}

25 Li F, Li S, Cheng T: TGF-beta1 promotes osteosarcoma cell migration and invasion through the miR-143versican pathway. Cell Physiol Biochem 2014;34:2169-2179.

26 Lin L, Tu HB, Wu L, Liu M, Jiang GN: MicroRNA-21 Regulates Non-Small Cell Lung Cancer Cell Invasion and Chemo-Sensitivity through SMAD7. Cell Physiol Biochem 2016;38:2152-2162.

27 Neerincx M, Sie DL, van de Wiel MA, van Grieken NC, Burggraaf JD, Dekker H, Eijk PP, Ylstra B, Verhoef C, Meijer GA, Buffart TE, Verheul HM: MiR expression profiles of paired primary colorectal cancer and metastases by next-generation sequencing. Oncogenesis 2015;4:e170.

28 Winter J, Jung S, Keller S, Gregory RI, Diederichs S: Many roads to maturity: microRNA biogenesis pathways and their regulation. Nat Cell Biol 2009;11:228-234.

29 Hata A, Kashima R: Dysregulation of microRNA biogenesis machinery in cancer. Crit Rev Biochem Mol Biol 2015;10.3109/10409238.2015.11170541-14.

30 Zheng J, Wu C, Xu Z, Xia P, Dong P, Chen B, Yu F: Hepatic stellate cell is activated by microRNA-181b via PTEN/Akt pathway. Mol Cell Biochem 2015;398:1-9.

31 Wang B, Li W, Guo K, Xiao Y, Wang Y, Fan J: miR-181b promotes hepatic stellate cells proliferation by targeting p27 and is elevated in the serum of cirrhosis patients. Biochem Biophys Res Commun 2012;421:4-8.

32 Wang B, Hsu SH, Majumder S, Kutay H, Huang W, Jacob ST, Ghoshal K: TGFbeta-mediated upregulation of hepatic miR-181b promotes hepatocarcinogenesis by targeting TIMP3. Oncogene 2010;29:1787-1797.

33 Kasuya A, Hoshino T, Aoshima M, Tatsuno K, Fujiyama T, Tokura Y: TGFbeta/SMAD4 signalling is inhibited in tumour cells and infiltrating lymphocytes of a patient with colon cancer-associated dermatomyositis. J Eur Acad Dermatol Venereol 2015;29:2265-2267.

34 Yang L, Yang J, Li J, Shen X, Le Y, Zhou C, Wang S, Zhang S, Xu D, Gong Z: MircoRNA-33a inhibits epithelial-tomesenchymal transition and metastasis and could be a prognostic marker in non-small cell lung cancer. Sci Rep 2015;5:13677.

35 Hurst DR, Edmonds MD, Welch DR: Metastamir: the field of metastasis-regulatory microRNA is spreading. Cancer Res 2009;69:7495-7498.

36 Wallis CJ, Gordanpour A, Bendavid JS, Sugar L, Nam RK, Seth A: MiR-182 Is Associated with Growth, Migration and Invasion in Prostate Cancer via Suppression of FOX01. J Cancer 2015;6:1295-1305.

37 Jiang J, Zheng X, Xu X, Zhou Q Yan H, Zhang X, Lu B, Wu C, Ju J: Prognostic significance of miR-181b and miR-21 in gastric cancer patients treated with S-1/Oxaliplatin or Doxifluridine/Oxaliplatin. PLoS One 2011;6:e23271.

38 Iliopoulos D, Jaeger SA, Hirsch HA, Bulyk ML, Struhl K: STAT3 activation of miR-21 and miR-181b-1 via PTEN and CYLD are part of the epigenetic switch linking inflammation to cancer. Mol Cell 2010;39:493506.

39 Chen L, Yang Q, Kong WQ, Liu T, Liu M, Li X, Tang H: MicroRNA-181b targets cAMP responsive element binding protein 1 in gastric adenocarcinomas. IUBMB Life 2012;64:628-635.

40 Zhu W, Shan X, Wang T, Shu Y, Liu P: miR-181b modulates multidrug resistance by targeting BCL2 in human cancer cell lines. Int J Cancer 2010;127:2520-2529.

41 Shen R, Wang Y, Wang CX, Yin M, Liu HL, Chen JP, Han JQ, Wang WB: MiRNA-155 mediates TAM resistance by modulating SOCS6-STAT3 signalling pathway in breast cancer. Am J Transl Res 2015;7:2115-2126.

42 Kong W, Yang H, He L, Zhao JJ, Coppola D, Dalton WS, Cheng JQ: MicroRNA-155 is regulated by the transforming growth factor beta/Smad pathway and contributes to epithelial cell plasticity by targeting RhoA. Mol Cell Biol 2008;28:6773-6784.

43 Davis BN, Hilyard AC, Lagna G, Hata A: SMAD proteins control DROSHA-mediated microRNA maturation. Nature 2008;454:56-61.

44 Yu Y, Ran Q: Nuclear SMAD2 restrains proliferation of glioblastoma. Cell Physiol Biochem 2015;35:17561763.

45 Wang L, Zhang X, Guo Y, Chen X, Li R, Liu L, Shi C, Guo C, Zhang Y: Involvement of BMPs/Smad signaling pathway in mechanical response in osteoblasts. Cell Physiol Biochem 2010;26:1093-1102.

46 Cai M, Wang Z, Zhang J, Zhou H, Jin L, Bai R, Weng Y: Adam17, a Target of Mir-326, Promotes Emt-Induced Cells Invasion in Lung Adenocarcinoma. Cell Physiol Biochem 2015;36:1175-1185. 